\title{
Epithelial to mesenchymal transition: The doorway to metastasis in human lung cancers
}

\author{
Chadrick E. Denlinger, MD, John S. Ikonomidis, MD, PhD, Carolyn E. Reed, MD, and \\ Francis G. Spinale, MD, PhD
}

Lung cancer remains a disease characterized by early metastasis and poor 5-year survival. Lung cancers are almost exclusively derived from epithelial tissues, and most tumor cells retain epithelial characteristics even as the tumor progresses. Small numbers of cells, however, are thought to undergo a process of epithelial to mesenchymal transition in which the malignant cells acquire a fibroblastlike morphology, lose intracellular adhesions, and become mobile. This process represents a crucial event in cancer invasion and metastasis. These mesenchymal cells may subsequently revert to an epithelial phenotype, allowing clinically relevant growth of metastases. Ongoing studies are required to determine ways in which the process of epithelial to mesenchymal transition can be exploited in patients with lung cancer for screening, diagnostic studies, and therapeutic options.

Lung cancer remains the most common cause of malignancy-related death among both men and women in America with more people dying of lung cancer than of melanoma, colorectal, breast, and prostate cancers combined. ${ }^{1}$ The overall 5-year survival among patients with non-small cell lung cancer (NSCLC) remains a dismal $15 \%$, primarily from development of distant metastatic disease. Despite the expectation that curative surgical resection of early stage NSCLC leaves patients disease free, $40 \%$ to $60 \%$ of these patients still have metastases develop and die of lung cancer. Efficacy of the best available chemotherapeutic agents to control metastasis has reached a plateau, and further advancement of cytotoxic therapy is unlikely. More recently, targeted agents have attracted interest after the introduction of antibodies and small molecule inhibitors of epithelial growth factor receptor and vascular endothelial growth factor. Novel agents delivered against these receptors provide survival benefits only among select patients, however, and the application of targeted agents to the overall population of patients with lung cancer provides only modest benefits. 2,3

Recently, the concept of epithelial to mesenchymal transition (EMT) has been proposed as a putative mechanism for

\footnotetext{
From Medical University of South Carolina and the Ralph H. Johnson VA Medical Center, Charleston, SC.

Received for publication Sept 28, 2009; revisions received Jan 10, 2010; accepted for publication Feb 1, 2010.

Address for reprints: Chadrick E. Denlinger, MD, 25 Courtenay Dr, Suite 7018, Charleston, SC 29425 (E-mail: denlinge@musc.edu).

J Thorac Cardiovasc Surg 2010;140:505-13

$0022-5223 / \$ 36.00$

Copyright (c) 2010 by The American Association for Thoracic Surgery doi:10.1016/j.jtcvs.2010.02.061
}

tumor invasion and metastasis in numerous types of solid tumors, including NSCLC, on the basis of clinical observations and laboratory investigations. ${ }^{4}$ EMT is a normal physiologic process that is essential for development as embryos progress from single-layered to multilayered organisms. EMT remains critical during development for the migration of some cells, such as neural crest cells, throughout the body. Even in adulthood, the induction of EMT remains a natural and necessary component of the inflammatory process and normal wound healing. ${ }^{5}$ The phenomenon of EMT has therefore been subdivided into 3 separate classes: class 1 EMT is involved with embryologic development, class 2 EMT is involved with wound healing, and class 3 EMT is involved with cancer cell invasion and migration.

Expression of mesenchymal markers among small clusters of cells within epithelium-derived tumors has been well described and is thought by some to represent a crucial event in malignancy that is required for local invasion and establishment of distant metastasis. ${ }^{6}$ Markers for EMT in human tissue specimens have been correlated with increased risk of cancer recurrence, the presence of metastasis, and decreased survival, supporting the concept that EMT events represent an aggressive biologic transition. This review provides an overview of our current understanding of the process of EMT in lung cancer and highlights the need for further investigations in this area by clinician-scientists. The goal is to identify ways in which EMT can be exploited for therapeutic benefits.

Evidence supporting the EMT hypothesis includes the observation of malignant cells with a mesenchymal phenotype in areas of carcinomas located at the advancing tumor front, where malignant cells infiltrate surrounding normal tissues. In addition, the ability to manipulate carcinoma cells in vitro into adopting mesenchymal phenotypes by the forced overexpression of EMT-linked transcription factors provides convincing evidence that EMT does occur. The clinical relevance of EMT in lung and other cancers is supported by the prognostic implications of mesenchymal markers within the primary tumor. Importantly, the presence of EMT cells within tumors has been correlated with the existence of metastasis, ${ }^{7}$ an increased risk of cancer recurrence, ${ }^{8}$ and decreased patient survival. ${ }^{9,10}$

Despite evidence supporting the hypothesis that EMT conveys enhanced invasiveness and metastasis, the concept remains controversial for several reasons. Most importantly, the EMT phenomenon does not conform to the canonical 


$\begin{array}{ll}\text { Abbreviations and Acronyms } \\ \text { E47 } & \text { basic helix-loop-helix transcription } \\ & \text { factor (E2A gene product) } \\ \text { ECM } & =\text { extracellular matrix } \\ \text { EMT } & =\text { epithelial to mesenchymal transition } \\ \text { GSK-3 } \beta & =\text { glycogen synthase kinase-3 } \beta \\ \text { IL } & =\text { interleukin } \\ \text { MET } & =\text { mesenchymal to epithelial transition } \\ \text { MMP } & =\text { matrix metalloproteinase } \\ \text { MT1-MMP }= & \text { membrane type 1 matrix } \\ & \text { metalloproteinase } \\ \text { NSCLC } & =\text { non-small cell lung cancer } \\ \text { SMAD } & =\text { family of proteins originally } \\ & \text { identified as class of proteins related } \\ & \text { to Sma and Mothers against decap- } \\ \text { TGF } \beta & \text { entaplegic protein } \\ & =\end{array}$

belief that cancers evolve by progressively acquiring genetic mutations that convey an increasingly aggressive phenotype. Instead, EMT events are transient changes driven by signals derived from the extracellular matrix (ECM) and inflammatory cells recruited to the advancing tumor front. Therefore, mesenchymal cells revert back to an epithelial form when signals driving the mesenchymal transition dissipate. The transient nature of EMT events makes scientific investigation of this phenomenon difficult for 2 reasons. First, transformed mesenchymal cells in human tumor specimens represent only a small percentage of the entire tumor, and therefore they can only be reliably identified by examining entire tissue slides, rather than representative samples in tissue microarrays. Similarly, because large segments of tumors do not uniformly undergo EMT, it is impossible to capture large numbers of transformed mesenchymal cells in vivo, as in frozen tumor samples, for investigation. Much of what has been learned about the EMT phenomenon has therefore come through in vitro investigations that have been correlated with clinical observations in tumor specimens.

\section{EMT AS A MARKER FOR POOR PROGNOSIS}

Malignant tumor cells with a mesenchymal phenotype appear clinically relevant, because the presence of EMT cells within primary tumors has been associated with worse clinical outcomes. The presence of transformed mesenchymal cells within tumors correlates with an increased risk of cancer recurrence and negatively relates to long-term survival among patients with numerous different types of solid tumor malignancies, including colorectal cancer, ${ }^{7}$ gastric cancer, ${ }^{11}$ and NSCLC, ${ }^{8,9}$ and is related to recurrent disease among patients with bladder cancer. ${ }^{8}$ Furthermore, expression of transcription factors driving the mesenchymal morphology has been linked to chemoresistance in pancreatic cell lines and in human specimens. ${ }^{12}$ In addition to its apparent effect on traditional cytotoxic agents, the mesenchymal phenotype also correlates with resistance to the novel epidermal growth factor receptor inhibitor erlotinib. ${ }^{13}$ This clinical information validates the understanding that EMT represents an aggressive transition of carcinomas into a more strongly pathologic phenotype.

The presence of EMT cells has also been associated with an increased rate of metastasis. In colorectal cancers, small clusters of EMT cells have been described as tumor budding that can be visualized penetrating normal surrounding tissues in histologic specimens. Tumor buds are highlighted with immunohistochemical stains for pancytokeratin. By means of receiver-operator curves, Prall and colleagues ${ }^{14}$ defined a cutoff value of 25 tumor buds/high-power field to discriminate a patient population with early-stage colorectal cancer at significantly greater risk for tumor metastasis and decreased survival than patients with fewer than 25 tumor buds/high-power field. This clinical observation further supports the concept that EMT contributes to tumor metastasis in patients with solid tumors.

Numerous biochemical markers for the induction of EMT have been described. The marker most tightly linked to EMT is the loss of E-cadherin expression, and the loss of this marker has been has been associated with an increased rate of metastasis among patients with breast cancer. ${ }^{15} \mathrm{Sim}$ ilarly, EMT markers have also been correlated with worse outcomes among patients with lung cancer. The expression in NSCLC tumor specimens of Slug, a transcription factor known to downregulate E-cadherin expression, correlates with an increased rate of cancer recurrence and decreased survival. ${ }^{10}$ Furthermore, forced overexpression of Slug in lung cancer cell lines used for the creation of xenografts in mice demonstrated markedly diminished E-cadherin expression, significantly enhanced expression of matrix metalloproteinase (MMP) 2, and enhanced propensity for metastases to develop in vivo. ${ }^{10}$ Collectively, these data support the concept that EMT events lead to tumor invasion and metastasis and decreased survival among patients with any of a multitude of solid tumor malignancies.

Currently, the working model suggests that EMT events allow malignant cells to disseminate throughout the body. The histology of established tumor metastases recapitulates the tissues of origin, however, rather than cells with a mesenchymal phenotype. Models of EMT as a prominent mechanism of metastasis suggest that migratory mesenchymal cells are able to revert back to an epithelial form through a process of mesenchymal to epithelial transition (MET), which could explain the epithelial morphology of cells in metastatic foci of lung cancers and other carcinomas (Figure 1). Ongoing studies will be required to determine whether the EMT cells are actually the cells responsible 


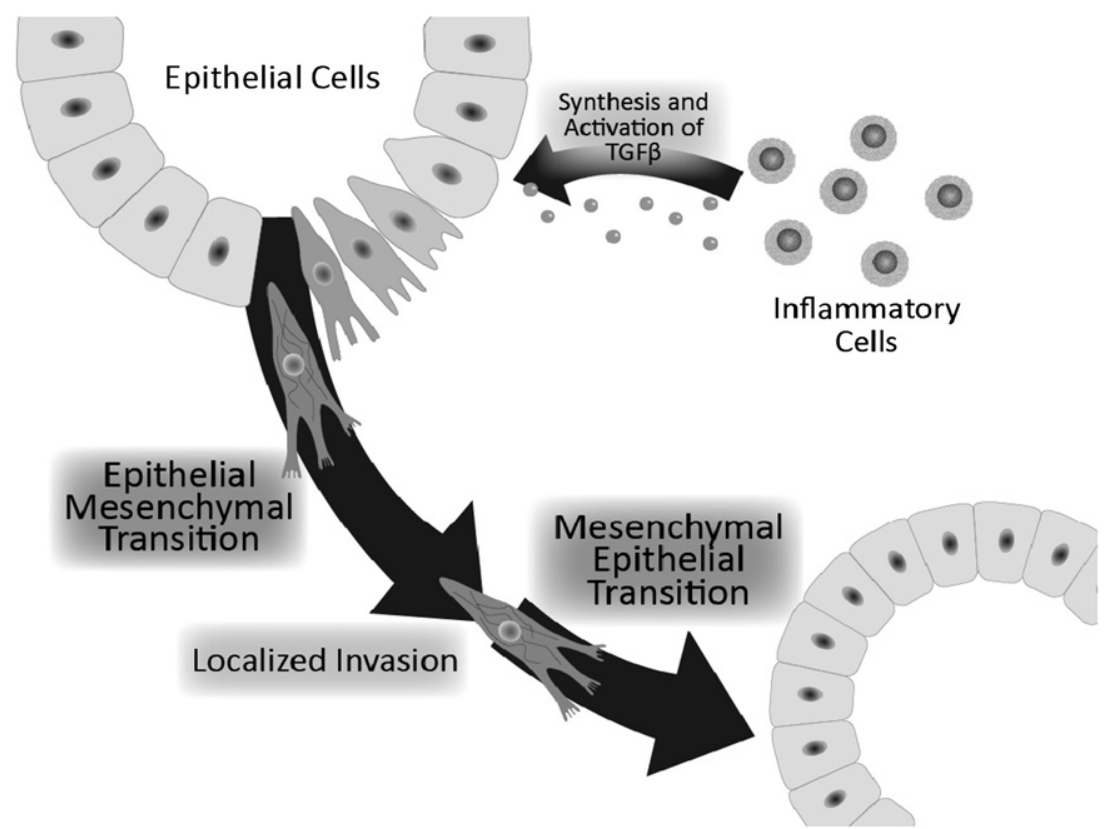

FIGURE 1. Local inflammatory cytokines at advancing tumor front play critical role in inducing biochemical and morphologic changes in epithelial tumors, leading to adoption of fibroblastlike mesenchymal form. Transformed mesenchymal cells lose intracellular adhesions and are capable of migrating through surrounding stroma. In remote locations, mesenchymal cells are thought to revert back to epithelial phenotype, which allows rapid growth and development of clinically significant metastases. $T G F \beta$, Transforming growth factor $\beta$.

for development of metastases or whether they are simply markers for an aggressive tumor that contains other components with the propensity to metastasize.

\section{EPITHELIAL TO MESENCHYMAL TRANSITION}

By definition, all carcinomas, including essentially all lung cancers, are derived from epithelial tissues. Even as bronchial epithelial cells become progressively dysplastic and transform into frankly invasive malignant cells, they retain morphologic and architectural characteristics of the epithelial tissues from which they arose. Most notably, epithelial cells retain cell polarity and dense cell-cell adherens junctions. In addition, adenocarcinomas continue to form glandular structures, and squamous cell carcinomas remain tightly clustered and often produce keratin pearls. Each of these features is consistent with the epithelial origins of carcinomas.

As tumors progress, clusters of transformed cells most frequently identified at the advancing tumor front undergo a morphologic transition whereby they lose epithelial characteristics and acquire a mesenchymal phenotype, recognizable by the overall cell appearance, intercellular relations, and biochemical changes. The transformed mesenchymal cells lose polarity, become elongated, and are less adherent to surrounding cells and the ECM. More importantly, mesenchymal cells also become motile, which facilitates invasion into local tissues and lymphovascular structures. In addition to gross morphologic changes in cells that have undergone EMT, several consistent molecular changes have been described (Figure 2).
Most notably, E-cadherin, which is a cell surface protein that is an essential component of adherens junctions between similar cells forming epithelial boundaries, is highly expressed on the cytoplasmic membrane of epithelial cells. E-cadherin expression is retained in carcinomas, including lung cancers, but its expression is lost in cells that have undergone EMT. In fact, E-cadherin loss in carcinoma cells is considered pathognomonic of the EMT phenomenon, and Ecadherin is considered to be a "master regulator" of EMT. ${ }^{16}$ Indeed, most signaling pathways involved in EMT converge on the regulation of E-cadherin expression. Not only is E-cadherin loss a marker for EMT, this molecular change also correlates with increased tumor grade, metastasis, and mortality. ${ }^{17}$ Despite tight associations of E-cadherin loss, the process of EMT, and poor patient outcomes, genetic mutations of E-cadherin are extremely rare in almost every type of carcinoma, with the exception of lobular breast carcinoma and diffuse gastric cancer. ${ }^{16}$ Instead, epigenetic modifications if the promoter region with hypermethylation are responsible for the transcriptional suppression of E-cadherin in EMT cells. Thus in EMT cells, E-cadherin genes are silenced by mechanisms that are completely reversible when driven by the appropriate stimuli.

A second protein, closely related to E-cadherin, that plays a functional role in EMT is $\beta$-catenin, which is an intracellular component of adherens junctions. In epithelial cells, $\beta$-catenin colocalizes with E-cadherin to the cytoplasmic membrane and is critical for linking intercellular adherens junctions with the intracellular actin cytoskeleton. As cells 


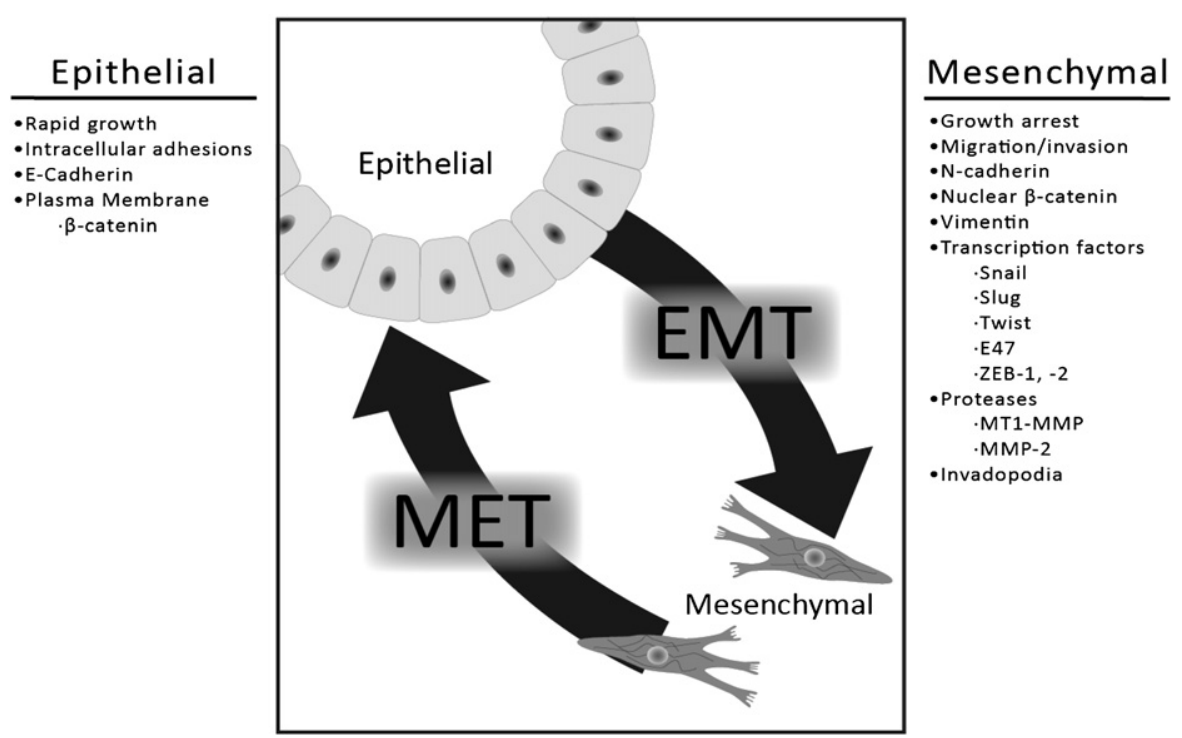

FIGURE 2. Interchangeable epithelial and mesenchymal phenotypes are characterized by several biochemical and cell surface protein markers that contribute to their respective morphologies and biologic activities. EMT, Epithelial to mesenchymal transition; ZEB, zinc finger E-box binding protein; $M E T$, mesenchymal to epithelial transition; $M T 1-M M P$, membrane type 1 matrix metalloproteinase; $M M P-2$, matrix metalloproteinase 2.

undergo EMT and E-cadherin expression is lost, $\beta$-catenin translocates from the cytoplasmic membrane to the nucleus, where it participates in transcriptional regulation of EMTrelated gene targets. Under normal circumstances, $\beta$-catenin released from the cytoplasmic membrane is rapidly phosphorylated by glycogen synthase kinase- $3 \beta$ (GSK- $3 \beta$ ), ubiquitinated, and degraded. Activation of the Wnt pathway, however, a common finding in cells that have undergone EMT, leads to GSK- $3 \beta$ inhibition, allowing $\beta$-catenin accumulation in the cytoplasm and nucleus. ${ }^{18}$ Nuclear $\beta$-catenin functions as a cofactor for T-cell factor, which drives transcription of genes required for EMT including Ets, Jun, Slug, matrilysin, fibronectin, and vimentin. ${ }^{19}$ Similar shuttling of $\beta$-catenin from the cytoplasmic membrane to the nucleus during the process of EMT has been described in numerous different solid tumors and cancer cell lines, including lung, breast, colon, pancreas, prostate, and gastric cancers. ${ }^{11}$ This conserved signaling pathway, present in numerous different carcinomas, provides some indication that $\beta$-catenin signaling plays a central role in EMT regulation (Figure 3).

In exchange for the loss of E-cadherin expression on the cytoplasmic membrane, mesenchymal cells express $\mathrm{N}$ cadherin, which is associated with migration, invasion, and metastasis. ${ }^{20} \mathrm{~N}$-cadherin drives expression of fibroblast growth factor receptors, leading to survival and resistance to apoptosis among cells that have become disassociated from the ECM. ${ }^{21}$ Other upregulated cell surface proteins that have been linked to transformed cells with a mesenchymal morphology include P-cadherin, cadherin-6, and carcinoembryonic antigen. $^{22}$
In addition to cell surface marker alterations consistent with the process of EMT, several transcription factors have also been tightly linked with EMT and are thought to play a role in its progression. Most notably, transcriptional repressors of E-cadherin have been isolated. These factors include the zinc finger proteins Snail and Slug, the 2-handed zinc E-box binding factors 1 and 2 , and the basic helixloop-helix factors E47 (E2A gene product) and Twist (transcription factor known to drive epithelial to mesenchymal transition and metastasis). Each of these factors has been associated with EMT in human tissue specimens, and causal roles for their activities in the process of EMT have been established in vitro. Forced overexpression of each of these transcription factors has been shown to induce EMT in cancer cell lines. A detailed analysis of Snail, Slug, and E47 expressions demonstrated that each of these transcription factors induced similar mesenchymal phenotypes even though the specific target genes activated or suppressed were significantly disparate, as determined by a gene Affymetrix array (Affymetrix, Inc, Santa Clara, Calif). ${ }^{23}$ Thus the presence of EMT cells within primary tumors of numerous different histologic types has been described and characterized by specific protein markers and regulators of gene transcription.

Although numerous characteristic of cells that have undergone EMT have been described, as outlined previously, mechanisms culminating in a switch from one metastable state to the next are less well understood. One postulated feed-forward loop contributing to EMT is outlined in Figure 3. This model requires simultaneous transforming growth factor $\beta$ (TGF $\beta$ ) signaling from local inflammatory cells, activation of the Wnt pathway, and cleavage of 


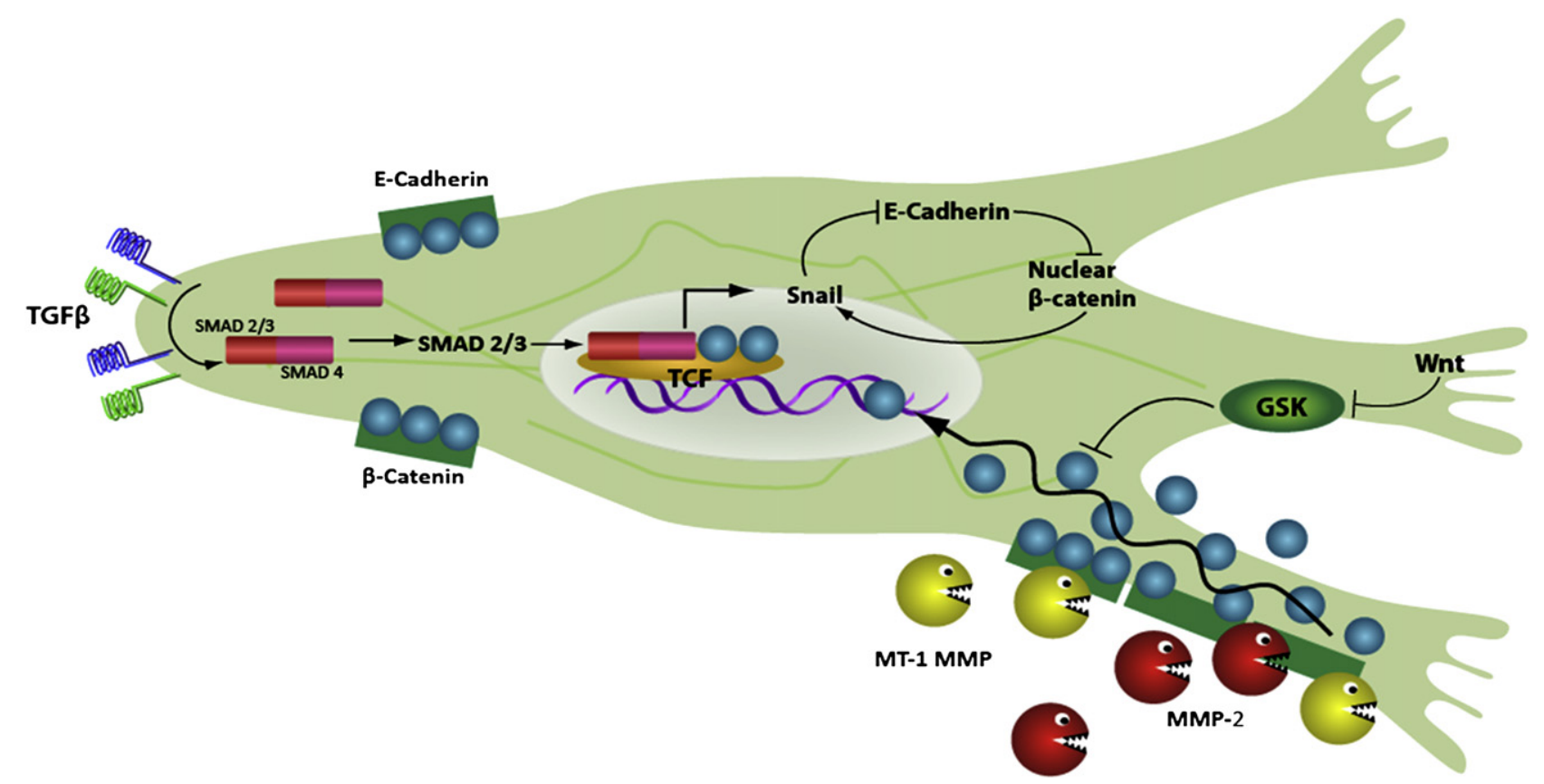

FIGURE 3. Proposed schematic of cellular and molecular events that can contribute to epithelial to mesenchymal transition. This schematic is not inclusive but attempts to integrate divergent pathways and processes presented in this review. Specifically, malignant cells retain epithelial characteristics until stimulated by tumor-infiltrating inflammatory cells driving release of transforming growth factor $\beta(T G F \beta)$ and interleukin 6 , which play causative role in conversion of cells from epithelial to mesenchymal phenotype. One feed-forward loop driving epithelial to mesenchymal transition process requires simultaneous transforming growth factor $\beta$ signaling, activation of Wnt pathway, and cleavage of intercellular adherens junctions by matrix metalloproteinase 2 (MMP-2, inset). Disruption of E-cadherin by matrix metalloproteinase 2 releases $\beta$-catenin into cytoplasm. Under normal circumstances, cytoplasmic $\beta$-catenin is rapidly phosphorylated by glycogen synthase kinase (GSK) $3 \beta$ and marked for ubiquitination and degradation. Activation of Wnt pathway inhibits glycogen synthase kinase $3 \beta$, allowing cytoplasmic and nuclear accumulation of $\beta$-catenin. Nuclear $\beta$-catenin acts as transcription cofactor for T-cell factor (TCF) in concert with SMAD 2 and 3 second messengers of transforming growth factor $\beta$ signaling. This transcription complex drives transcription of several epithelial to mesenchymal transition-related genes, including transcription factor Snail. Primary target for Snail is transcriptional suppression of E-cadherin, leading to further nuclear accumulation of $\beta$-catenin, thus completing feed-forward loop and stabilizing cell in mesenchymal form.

intercellular adherens junctions by MMP2. The disruption of E-cadherin by MMP2 releases $\beta$-catenin into the cytoplasm. Under normal circumstances, cytoplasmic $\beta$-catenin is rapidly phosphorylated by GSK-3 $\beta$ and marked for ubiquitination and degradation. Activation of the Wnt pathway inhibits GSK- $3 \beta$, allowing cytoplasmic and nuclear accumulation of $\beta$-catenin. Nuclear $\beta$-catenin acts as a transcription cofactor for T-cell factor, in concert with the SMAD (a family of proteins involved in transforming growth factor signaling, originally identified as class of proteins related to Sma and Mothers against decapentaplegic protein) 2 and 3 second messengers of TGF $\beta$ signaling. This transcription complex promotes transcription of several EMT-related genes including vimentin, fibronectin, and the EMT -promoting transcription factor Snail. A primary target for Snail is the transcriptional suppression of E-cadherin, thus completing the feed-forward loop and stabilizing the cell in a mesenchymal form.

\section{Local Inflammatory Induction of EMT}

Our current understanding of EMT in transformed cells is that this process is driven by local inflammatory cytokines.
Evidence supporting this phenomenon has been demonstrated in human tumor specimens as well as in vitro investigations. Tumor budding or clusters of EMT cells are typically located in regions of tumors associated with the intense recruitment of inflammatory cells. ${ }^{24}$ Importantly, both the presence of tumor-infiltrating macrophages and TGF $\beta$ have been associated with worse outcomes for patients with NSCLC, ${ }^{25}$ with these correlations most pronounced among patients with adenocarcinoma. This finding suggests that interactions between the advancing tumor cells and local inflammatory cells may play a role in the induction of EMT.

$\operatorname{TGF} \beta$ signaling, which has been implicated as a major contributor to EMT among malignant cells at the advancing front of tumors, is mediated through TGF $\beta$ type I and type II transmembrane receptors. The type I and type II TGF $\beta$ receptors form heterodimeric complexes that activate the downstream second messengers SMAD 2 and 3, which form complexes with SMAD 4. The SMAD 2/3-4 complex activates the T-cell factor transcription factor, which is tightly related to the EMT induction when bound to $\beta$-catenin and SMAD. ${ }^{26}$ Cellular responses to $\mathrm{TGF} \beta$ signaling among transformed and normal cells depends greatly on 
the expressions of various SMAD secondary messengers and available transcription cofactors, which couple with the SMAD cofactors at the time of TGF $\beta$ exposure. In transformed cells, TGF $\beta$ binds with SMAD 2 or 3 and 4, which couple with the transcription factor Snail. This leads to transcriptional repression of E-cadherin and the Coxsackie and adenovirus receptor, which are both critical components of epithelial cell tight junctions that are downregulated in human cancer cells by TGF $\beta$-induced EMT events. ${ }^{27}$ In addition, TGF $\beta$ signaling may also induce EMT events mediated through type I receptors independent of SMAD secondary messengers. Under these circumstances, the TGF $\beta$ receptors activate any of several pathways, including mitogenactivated protein kinase and phosphoinositide 3 kinase, both of which are linked with tumor progression. ${ }^{28,29}$

In addition to TGF $\beta$, another putative inflammatory signaling molecule implicated in the induction of EMT in malignancies is interleukin (IL) 6. This signaling molecule suppresses E-cadherin in breast cancer cells. Furthermore, cell lines that endogenously express high levels of IL-6 have been shown to maintain a mesenchymal phenotype characterized by low E-cadherin expression and constitutively high levels of vimentin, N-cadherin, Snail, and Twist. ${ }^{30}$ Moreover, elevated serum levels of IL-6 correlate with advanced tumor stage, number of metastases, and overall poor survival among patients with breast cancer. ${ }^{31}$

Recently, circulating serum levels of inflammatory cytokines have also been linked to survival among patients with lung cancer. ${ }^{32}$ Specific cytokines most closely tied to survival have varied according to the ethnic backgrounds of patients with cancer. Specifically, African American and white patients with lung cancer had worse survival rates associated with elevated IL-6 levels. In addition, serum levels of IL-10 and IL-12 both correlated inversely with patient survival in the African American population. ${ }^{32}$ Others have also noted the prognostic value of inflammatory cytokines. Expressions of IL-6, IL-8, and IL-10 in uninvolved lung tissue of patients with NSCLC have been associated with increased risk of lymph node metastasis. ${ }^{33}$ Circulating serum levels of cytokines implicated in EMT induction in NSCLC and other solid tumors therefore also portend worse long-term clinical outcomes. Although some understanding of the role of inflammatory mediators in EMT exists, these interactions need to be further elucidated.

\section{Invadopodia, Podosomes, and Dorsal Ruffles}

It is recognized that transformed mesenchymal cells facilitate local invasion and distant metastasis through a process that is initiated by degrading the integrity of the basement membrane, which relates to the local MMP expression. Retrospective analyses from patients with cancer have identified that expression of MMPs, such as membrane-type $1 \mathrm{MMP}$ (MT1-MMP), MMP-1, MMP-2, MMP-3, MMP-7, and MMP-13, correlate with tumor progression and metastasis. ${ }^{34}$
Less is understood, however, about the mechanisms through which local inflammation and the induction of EMT contribute to altered interactions between tumor cells and the ECM that allow matrix degradation and motility of transformed cells. Recently, putative mechanisms for this process have been proposed and supported by direct observations of tumors and experimental modeling.

Inherently mobile cells, such as macrophages and osteoclasts, contain various cytoplasmic membrane processes bound to the intracellular actin skeleton called podosomes, invadopodia, and dorsal ruffles. ${ }^{35}$ Interestingly, similar cellular processes have been identified on motile EMT tumor cells in the setting of local inflammation. ${ }^{36}$ Podosomes and invadopodia are similar to focal adhesion in that they possess transmembrane complexes that interact with surrounding cells and the intracellular matrix. They differ, however, in that they are far more dynamic and constantly recycle as cells migrate. ${ }^{35}$ Podosomes are rich in the structural proteins $\mathrm{F}$-actin, vinculin, $\alpha$-actinin, and Src, which maintain the podosome shape and interaction with the actin cytoskeleton. ${ }^{35}$ Podosomes also, however, contain proteins that regulate actin polymerization such as the actinsevering and -capping protein gelsolin, the Arp $2 / 3$ complex, $\mathrm{N}-\mathrm{WASP}$, and the actin-bundling protein fimbrin. The concentration of actin regulatory proteins within podosomes reflects the continual remodeling of these structures. ${ }^{35} \mathrm{By}$ way of contrast, more stable focal adhesions lack these actin-restructuring protein complexes.

Invadopodia are structurally similar to podosomes but demonstrate features that more clearly define their role related to ECM degradation and cell migration. ${ }^{37}$ Invadopodia were first identified at the exact locations where ECM degradation was occurring. Later, it was discovered that they contain an enriched presence of MMPs responsible for ECM degradation as well as modulating extracellular signaling cascades. ${ }^{38}$ Specifically, MT1-MMP localizes to invadopodia, where it is an essential component and appears to play a pivotal role in pathologic cell invasion and migration. ${ }^{39}$ MT1-MMP activity has also been found to be essential for in vitro migration of cells through a Matrigel (BD Biosciences, San Jose, Calif) matrix. ${ }^{40}$ Although MT1-MMP is normally expressed in cells of mesenchymal origin, including fibroblasts, muscular cells, and osteoblasts, this protein is also expressed on cells from numerous epithelium-based solid tumors. Experimental in vitro studies have shown that interference with either the MMP activity or actin remodeling of invadopodia suppresses ECM degradation. This information suggest a critical role for invadopodia in contributing to characteristics and behavior consistent with the EMT phenotype.

MT1-MMP expression was initially reported in lung cancer cells and fibroblasts adjacent to the advancing tumor front. ${ }^{41}$ More recent studies have confirmed this finding and also identified the accumulation of MMP- 2 at advancing 
tumor fronts. ${ }^{42}$ Similar patterns of MT1-MMP expression have also been described in other carcinomas, including gastric, colon, liver, breast, bladder, thyroid, head and neck, ovarian, and cervical cancers. One of the primary functions of MT1-MMP, in addition to ECM degradation, is the cleavage and activation of the MMP-2 precursor. The expressions of both MT1-MMP and MMP-2 correlate with poor prognosis and have been postulated to result from Wnt signaling, which is also an upstream activator of EMT. ${ }^{39}$ Strong supporting data for the development of invadopodia in cells that have undergone EMT has been found in squamous cell carcinomas of the head and neck. ${ }^{43}$ These tumor specimens demonstrate an exchange of podosome structures in noninvasive tumors for invadopodia in the invasive components of tumors where EMT has occurred. Collectively, these data support the role of invadopodia and podosomes in contributing to the activity and behavior of EMT cells in cancer.

\section{Growth Arrest}

Although the presence of EMT foci within primary tumors is indicative of poor outcomes, EMT cells are not particularly aggressive while in the mesenchymal state. ${ }^{44}$ In fact, EMT cells undergo cell cycle arrest. Transcription factors associated with EMT influence expression of numerous genes. Although EMT-related transcription factors were initially thought to primarily silence gene expression, significantly more genes appear to be directly activated than inhibited by these factors. The first recognized targets of transcriptional suppression in EMT cells were epithelial cell surface markers; however, EMT-associated transcription factors have been shown to upregulate transcription of numerous factors involved in cell cycle regulation. In fact, nearly a fourth of all genes regulated by Snail, Slug, and E47 are involved in cell cycle progression. ${ }^{23}$ Included among this list of genes activated during EMT are the cell cycle checkpoint proteins $\mathrm{p} 21$ and $\mathrm{p} 15 .^{45}$

Growth arrest among transformed mesenchymal cells is problematic from a treatment standpoint for 2 reasons. Most importantly, growth arrested mesenchymal cells are inherently resistant to the cytotoxic effects of chemotherapy designed to kill rapidly dividing cells. Thus the EMT cell population may represent "stem cells" that can repopulate tumors, leading to cancer recurrence after treatment. Furthermore, because the EMT phenotype is completely reversible, cells are able to revert back to an epithelial form characterized by rapid growth. EMT cells therefore represent the cancer's ability to recur.

\section{MESENCHYMAL TO EPITHELIAL TRANSITION}

Micrometastatic mesenchymal cell colonies revert back to an epithelial phenotype through a process of MET as the result of poorly elucidated stimuli that cause them to begin to grow rapidly, invade surrounding tissues, and ultimately lead to the patient's death. ${ }^{46}$ Determination of mechanisms responsible for MET is clinically relevant because this process appears crucial for micrometastases to develop into significant disease. In vitro studies of colon cancer cell lines provide some evidence that the MET phenomenon may result from enhanced cell density and paracrine stimulation from neighboring cells. ${ }^{47}$ Patient-based data are lacking, however, simply because MET events are likely to occur in very small foci of metastatic disease that have yet to become clinically detectable. Once a metastasis has achieved a size sufficient for clinical recognition, the vast majority of cells populating the metastasis are already in the epithelial form, and MET has already occurred.

Widely disseminated cancer cells have been identified in the blood and bone marrow of patients with breast cancer without clinical evidence of metastatic disease, and in some patients these disseminated tumor cells may remain dormant throughout the patient's life span. ${ }^{48}$ Recent studies have also identified disseminated tumor cells in the bone marrow of patients with NSCLC. Moreover, genomic changes present within disseminated tumor cells were consistent with mutations identified in brain metastases of the same patients, suggesting that a similar population of disseminated cells was present in both the bone marrow and brain tissue, leading to appreciable brain metastases. ${ }^{49}$ These observations provide convincing evidence that subclinical micrometastases can in fact develop into clinically significant metastatic disease. It is therefore conceivable that NSCLC cells disseminate in a mesenchymal form and subsequently reactivate with an epithelial phenotype, leading to metastatic growth.

Ongoing investigations are required to determine the circumstances under which MET occurs in vitro and whether this process plays a meaningful role in the establishment of metastatic disease in patients with NSCLC and other cancers. An important clinical question is whether stimuli leading to MET events can be arrested either pharmacologically or with antibodies targeting necessary elements of the MET process.

\section{SUMMARY}

Substantial evidence supports the phenomenon of EMT existing in numerous types of carcinomas, including lung cancer. Furthermore, correlative data support the hypothesis that EMT represents a critical event in tumor progression, despite the fact that the EMT cells are not particularly aggressive while in the mesenchymal state. Ongoing studies will be required to further elucidate the role of EMT in the invasion and metastasis of lung and other cancers. In addition, ongoing investigations will determine how the presence of transformed mesenchymal cells can be exploited for diagnostic and treatment purposes in patients with lung cancer. Currently, investigations related to delivering targeted therapy toward markers for EMT are only in the 
preclinical stages of development. Development of strategies that target this cell population may contribute significantly to our lung cancer treatment armamentarium, however, because EMT creates a relatively unique cell population in the adult patient. Furthermore, understanding the mesenchymal component of epithelium-derived tumors is important because these cells may represent a chemoresistant component with the potential to both repopulate the primary tumor and metastasize after treatment. Certainly, collaborative investigations between clinicians and basic science investigators are required to establish a better understanding of the process of EMT and how it relates to the progression of lung cancer, as well as other solid tumor malignancies.

We thank Kaeyln Hawkins for her contributions in creating and editing Figure 1 of this article.

\section{References}

1. Jemal A, Siegel R, Ward E, Hao Y, Xu J, Murray T, et al. Cancer statistics, 2008. CA Cancer J Clin. 2008;58:71-96.

2. Sandler A, Gray R, Perry MC, Brahmer J, Schiller JH, Dowlati A, et al. Paclitaxelcarboplatin alone or with bevacizumab for non-small cell lung cancer. $N$ Engl J Med. 2006;355:2542-50. Erratum in: N Engl J Med. 2007;356:318.

3. Joy AA, Butts CA. Extending outcomes: epidermal growth factor receptortargeted monoclonal antibodies in non-small cell lung cancer. Clin Lung Cancer. 2009;1010(Suppl. 1):S24-9.

4. Brabletz T, Jung A, Spaderna S, Hlubek F, Kirchner T. Opinion: migrating cancer stem cells-an integrated concept of malignant tumor progression. Nat Rev Cancer. 2005;5:744-9.

5. Voulgari A, Pintzas A. Epithelial-mesenchymal transition in cancer metastasis: mechanisms, markers and strategies to overcome drug resistance in the clinic. Biochim Biophys Acta. 2009;1796:75-90.

6. Thiery J. Epithelial-mesenchymal transition in development and pathogenesis. Curr Opin Cell Biol. 2003;15:740-6.

7. Ueno H, Murphy J, Jass JR, Mochizuki H, Talbot IC. Tumor 'budding' as an index to estimate the potential of aggressiveness in rectal cancer. Histopathology. 2002;40:127-32.

8. Bruyere F, Namdarian B, Corcoran NM, Pedersen J, Ockrim J, Voelzke BB, et al. Snail expression is an independent predictor of tumor recurrence in superficial bladder cancers. Urol Oncol. Epub 2009 Jan 20.

9. Soltermann A, Tischler V, Arbogast S, Braun J, Probst-Hensch N, Weder W, et al. Prognostic significance of epithelial-mesenchymal and mesenchymal-epithelial transition protein expression in non-small cell lung cancer. Clin Cancer Res. 2008; $14: 7430-7$.

10. Shih J. Transcription repressor slug promotes carcinoma and predicts outcome of patients with lung adenocarcinoma. Clin Cancer Res. 2005;11:8070-8.

11. Kim MA, Lee HS, Lee HE, Kim JH, Yang HK, Kim WH. Prognostic importance of epithelial-mesenchymal-related protein expression in gastric carcinoma. Histopathology. 2009;54:442-51.

12. Arumugam T, Ramachandran V, Fournier KF, Wang H, Marquis L, Abbruzzese JL, et al. Epithelial to mesenchymal transition contributes to drug resistance in pancreatic cancer. Cancer Res. 2009;69:5820-8.

13. Haddad Y, Choi W, McConkey DJ. Delta-crystallin enhancer binding factor 1 controls the epithelial to mesenchymal transition phenotype and resistance to the epithelial growth factor receptor inhibitor erlotinib in human head and neck squamous cell carcinoma lines. Clin Cancer Res. 2009;15:532-42.

14. Prall F, Nizze H, Barten M. Tumor budding as prognostic factor in stage I/II colorectal carcinoma. Histopathology. 2005;47:17-24.

15. Oka H, Shiozaki H, Kobayashi K, Inoue M, Tahara H, Kobayashi T, et al. Expression of E-cadherin cell adhesion molecules in human breast cancer tissues and its relationship to metastasis. Cancer Res. 1993;53:1696-701.

16. Guarino M, Rubino B, Ballabia G. The role of epithelial-mesenchymal transition in cancer pathology. Pathology. 2007;39:305-18.

17. Hirohashi S. Inactivation of the E-cadherin-mediated cell adhesion system in human cancers. Am J Pathol. 1998;153:333-9.
18. Nelson WJ, Nusse R. Convergence of Wnt, $\beta$-catenin, and cadherin pathways. Science. 2004;303:1483-7.

19. Connacci-Sorrell M, Simcha I, Ben-Yedidia T, Blechman J, Savagner P, BenZe'ev A, et al. Autoregulation of E-cadherin expression by cadherin-cadherin interactions: the roles of $\beta$-catenin signaling, Slug, and MAPK. J Cell Biol. 2003; 163:847-57.

20. Tomita K, van Bokhoven A, van Leenders GJ, Ruijter ET, Jansen CF, Bussemakers MJ, et al. Cadherin switching in human prostate cancer progression. Cancer Res. 2000;60:3650-4.

21. Suyama K, Shapiro I, Guttman M, Hazan RB. A signaling pathway leading to metastasis is controlled by N-cadherin and the FGF receptor. Cancer Cell. 2002;2: 301-13.

22. Schmidmaier R, Baumann P. Anti-adhesion evolves to a promising therapeutic concept in oncology. Curr Med Chem. 2008;15:978-90.

23. Moreno-Bueno G, Cubillo E, Sarrió D, Peinado H, Rodríguez-Pinilla SM, et al. Genetic profiling of epithelial cells expressing E-cadherin repressors reveals a distinct role for Snail, Slug, and E47 factors in epithelial-mesenchymal transition. Cancer Res. 2006;66:9543-56.

24. Goede V, Brogelli L, Ziche M, Augustin HG. Induction of inflammatory angiogenesis by monocyte chemoattractant protein-1. Int J Cancer. 1999;82:765-70.

25. Hasegawa Y, Takanashi S, Kanehira Y, Tsushima T, Imai T, Okumura K. Transforming growth factor- $\beta 1$ level correlates with angiogenesis tumor progression, and prognosis in patients with nonsmall cell lung carcinoma. Cancer. 2001;91:964-71.

26. Nawshad A, Lagamba D, Polad A, Hay ED. Transforming growth factor-beta signaling during epithelial-mesenchymal transformation: implications for embryogenesis and tumor metastasis. Cells Tissues Organs. 2005;179:11-23.

27. Lacher MD, Tiirikainen MI, Saunier EF, Christian C, Anders M, Oft M, et al. Transforming growth factor $-\beta$ receptor inhibition enhances adenoviral infectability of carcinoma cells via upregulation of Coxsackie and adenovirus receptor in conjunction with reversal of epithelial-mesenchymal transition. Cancer Res. 2006;66:1648-57.

28. Yu L, Hebert MD, Zhang YE. TGF-beta receptor-activated p38 MAP kinase mediates Smad-independent TGF-beta responses. EMBO J. 2002;21:3749-59.

29. Bhowmick NA, Ghiassi M, Bakin A, Aakre M, Lundquist CA, Engel ME, et al. Transforming factor- $\beta 1$ mediates epithelial to mesenchymal transdifferentiation through a RhoA-dependant mechanism. Mol Biol Cell. 2001;12:27-36.

30. Sullivan NJ, Sasser AK, Axel AE, Vesuna F, Raman V, Ramirez N, et al. Interleukin-6 induces an epithelial-mesenchymal transition phenotype in human breast cancer cells. Oncogene. 2009;28(33):2940-7.

31. Salgado R, Junius S, Benoy I, Van Dam P, Vermeulen P, Van Marck E, et al. Circulating interleukin-6 predicts survival in patients with metastatic breast cancer. Int J Cancer. 2003;103:642-6.

32. Enewold L, Mechanic LE, Bowman ED, Zheng YL, Yu Z, Trivers G, et al. Serum concentrations of cytokines and lung cancer survival in African Americans and Caucasians. Cancer Epidemiol Biomarkers Prev. 2009;18:215-22.

33. Seike M, Yanaihara N, Bowman ED, Zanetti KA, Budhu A, Kumamoto K, et al. Use of a cytokine gene expression signature in lung adenocarcinoma and the surrounding tissue as a prognostic classifier. J Natl Cancer Inst. 2007;99:1257-69.

34. Duryuginia EI, Quigley JP. Matrix metalloproteinase and tumor metastasis. Cancer Metastasis. 2006;25:9-34.

35. Buccione R, Orth JD, McNiven MA. Foot and mouth: podosomes, invadopodia and circular dorsal ruffles. Nature Rev Mol Cell Biol. 2004;5:647-57.

36. Mandal S, Johnson KR, Wheelock MJ. TGF- $\beta$ induces formation of F-actin cores and matrix degradation in human breast cancer cells via distinct signaling pathways. Exp Cell Res. 2008;314:3478-93.

37. Basbaum CB, Werb Z. Focalized proteolysis: spatial and temporal regulation of extracellular matrix degradation at the cell surface. Curr Opin Cell Biol. 1996; 8:731-8.

38. Chen WT. Proteolytic activity of specialized surface protrusions formed at rosette contact sites of transformed cells. J Exp Zool. 1989;251:167-85.

39. Seiki M. Membrane-type 1 matrix metalloproteinase: a key enzyme for tumor invasion. Cancer Letter. 2003;194:1-11.

40. Ueda J, Kajita M, Suenaga N, Jugii K, Seika M. Sequence-specific silencing of MT1-MMP expression suppresses tumor cell migration and invasion: importance of MT1-MMP as a therapeutic target for invasive tumors. Oncogene. 2003;22: 8716-22.

41. Sato H, Takino Y, Okada J, Cao J, Shinagawa A, Yamamoto E, et al. A matrix metalloproteinase expressed on the surface of invasive tumor cells. Nature. 1994;370:61-5.

42. Hofmann UB, Eggert AA, Blass K, Brocker EB, Becker JC. Expression of matrix metalloproteinases in the microenvironment of spontaneous and experimental 
melanoma metastases reflects the requirements for tumor formation. Cancer Res. 2003;63:8221-5.

43. Takkunen M, Hukkanen M, Liljeström M, Grenman R, Virtanen I. Podosome-like structures of non-invasive carcinoma cells are replaced in epithelial-mesenchymal transition by actin comet-embedded invadopodia. J Cell Mol Med. Epub 2009 Jul 28.

44. Jung A, Schrauder M, Oswald U, Knoll C, Sellberg P, Palmqvist R, et al. The invasion front of human colorectal adenocarcinomas shows co-localization of nuclear $\beta$-catenin, cyclin D1, and p16INK4A and is a region of low proliferation. Am J Pathol. 2001;159:1613-7.

45. Hu CT, Wu JR, Cheng CC, Cheng CC, Wu WS. The transcriptional factor Snail simultaneously triggers cell cycle arrest and migration of human hepatoma HepG2. J Biomed Sci. 2008;15:343-55.
46. Barker N, Clevers H. Tumor environment: a potent driving force in colorectal cancer? Trends Mol Med. 2001;7:535-7.

47. Brabletz T, Jung A, Reu S, Porzner M, Hlubek F, Kunz-Schughart AL, et al. Variable $\beta$-catenin expression in colorectal cancers indicates tumor progression driven by the tumor environment. Proc Natl Acad Sci. 2001;98:10356-61.

48. Bidard FC, Vincent-Salomon A, Sigal-Zafrani B, Rodrigues M, Diéras V Mignot L, et al. Time to metastatic relapse and breast cancer cells dissemination in bone marrow at metastatic relapse. Clin Exp Metastasis. 2008;25: 871-5.

49. Wrage M, Rousaari S, Eijk PP, Kaifi JT, Hollmén J, Yekebas EF, et al. Genomic profiles associated with early micrometastasis in lung cancer: relevance of $4 \mathrm{q}$ deletion. Clin Cancer Res. 2009;15:1566-74 